\title{
ESTRATEGIAS DE MANEJO DURANTE EL CLIMATERIO Y MENOPAUSIA. GUÍA PRÁCTICA DE ATENCIÓN RÁPIDA
}

\author{
Félix Dasio Ayala Peralta ${ }^{1, a}$
}

\begin{abstract}
RESUMEN
El climaterio es etapa de vida fisiológica de la mujer donde se presentan síntomas climatéricos y aparición de enfermedades crónicas propias de este periodo, que afectan la calidad de vida relacionada a la salud femenina. La clínica durante el climaterio es variable; pero, los síntomas vasomotores y el síndrome genitourinario de la menopausia son síntomas cardinales de la menopausia.

Las estrategias en el manejo del climaterio han cambiado en los últimos años a raíz de resultados de recientes investigaciones en esta área, ya que no solo se utilizan terapia hormonal y no hormonal; sino que las modificaciones en el estilo de vida, incluida la dieta y el ejercicio, pueden ayudar a reducir los síntomas de la menopausia. Las indicaciones de terapia hormonal de la menopausia están circunscritas para manejo de síntomas climatéricos que afectan la calidad de vida en forma severa, tratamiento de osteoporosis posmenopáusica o riesgo de osteoporosis, menopausia prematura y síndrome genitourinario de la menopausia. Otras medidas terapéuticas deben ser utilizadas en forma individualizada según la necesidad de cada paciente.
\end{abstract}

Palabras clave: Climaterio; Menopausia; Terapia hormonal; Calidad de vida (Fuente: DeCS BIREME).

\section{MANAGEMENT STRATEGIES DURING CLIMATE AND MENOPAUSE. PRACTICAL GUIDE TO QUICK ATTENTION}

\begin{abstract}
The climacteric is a stage of a woman's physiological life where climacteric symptoms and the appearance of chronic diseases typical of this period appear, which affect the quality of life related to female health. The clinic during the climacteric is variable; but, vasomotor symptoms and genitourinary menopause syndrome are cardinal symptoms of menopause.

The strategies in the management of the climacteric have changed in the last years due to the results of recent investigations in this area, since not only are hormonal and non-hormonal therapy used; Instead, lifestyle modifications, including diet and exercise, can help reduce menopausal symptoms. The indications for menopausal hormone therapy are circumscribed for the management of climacteric symptoms that severely affect quality of life, treatment of postmenopausal osteoporosis or risk of osteoporosis, premature menopause, and genitourinary syndrome of menopause. Other therapeutic measures must be used individually according to the needs of each patient.
\end{abstract}

Key words: Climacteric; Menopause; Hormonal therapy; Quality of life (Source: MeSH NLM).

\section{INTRODUCCIÓN}

Las manifestaciones clínicas durante el climaterio son variables; pero, los síntomas vasomotores (SVM) y el síndrome genitourinario de la menopausia (SGM) son síntomas cardinales de la menopausia ${ }^{1,2}$.

Las mujeres comienzan un cese gradual de la fertilidad alrededor de los 35 años, seguido de un período aproximado de 10 años hasta llegar a la pérdida completa de la actividad folicular, presentándose la menopausia entre los 50 y 52 años de edad ${ }^{3-6}$.

Se han reportado que 8 de cada 10 mujeres tiene al menos uno de los síntomas clásicos (físicos o emocionales) durante o posterior al climaterio, de las cuales el $45 \%$ considera que impactan de manera negativa en su calidad de $\operatorname{vida}^{3,7,8}$.

La mujer y el médico tratante se enfrentan con varias decisiones importantes como, si se debe empezar o no la terapia hormonal de la menopausia (THM), siendo cada vez más frecuentes los estudios que incorporan las escalas de la calidad de vida como instrumento específico para valorar la acción de una intervención farmacológica ${ }^{3,9-11}$.

De ahí la importancia de la denominada "hipótesis de tiempo" o "ventana de oportunidad" que sugieren que el uso de terapia hormonal de menopausia (THM) en mujeres más jóvenes tiene efectos beneficiosos sobre

\footnotetext{
Universidad Nacional Mayor de San Marcos.

a Profesor ordinario. Departamento Académico de Ginecología y Obstetricia. Jefe de Servicio de Climaterio y Menopausia del Instituto Nacional Materno Perinatal. Lima,Perú.

Citar como: Ayala-Peralta FD. Estrategias de manejo durante el climaterio y menopausia. Guía práctica de atención rápida. Rev Peru Investig Matern Perinat 2020; 9(2): 34-41

DOI https://doi.org/10.33421/inmp.2020204
}

Recibido: 22-04-20 Aprobado: 30-06-20 
los resultados de salud que no se observan en mujeres mayores que comienzan la THM muchos años después de la menopausia ${ }^{12}(10)$. Existe consenso global de todas las principales sociedades científicas de menopausia de que hay evidencias de que la dosis estándar de THM con estrógeno solo puede disminuir la enfermedad coronaria y mortalidad por todas las causas en mujeres menores de 60 años y dentro de los 10 años de la menopausia; inclusive, podrían reducir la progresión de la aterosclerosis temprana, la enfermedad coronaria y la muerte por cualquiera causa ${ }^{13}$.

Cabe destacar que la THM, en la actualidad, sigue siendo la estrategia útil en el manejo durante el climaterio, sopesando los beneficios y los riesgos que conlleva dicho tratamiento ${ }^{14,15}$.

Finalmente señalar que, la THM y los cambios en los estilos de vida saludables son medidas efectivas para mejorar la calidad de vida durante el climaterio ${ }^{1,2,16}$.

\section{TERMINOLOGÍA UTILIZADA EN CLIMATERIO}

Climaterio. Es la etapa en la vida de la mujer que se inicia con la declinación de la función ovárica hasta la ausencia total de secreción estrogénica por el ovario, que conllevan a un déficit hormonal con o sin sintomatología y/o riesgos variables. La Federación Internacional de Ginecología y Obstetricia (FIGO), define como el período de tiempo que se extiende desde el inicio de la declinación de la madurez reproductiva hasta el inicio de la senectud. Se inicia a los 35 años de edad y finaliza a los 65 años de edad, que marca el comienzo de la senectud ${ }^{17-23}$.

Síndrome Climatérico. Conjunto de síntomas y signos que anteceden y siguen a la menopausia, como consecuencia de la declinación o cese de la función ovárica ${ }^{21}$.

Menopausia Natural o Espontánea. Es el momento en que ocurre la última hemorragia uterina menstrual espontánea debido al cese definitivo de la actividad folicular ovárica; y se establece como diagnóstico retrospectivamente, cuando han transcurrido un período de amenorrea de seis meses (FIGO) o de doce meses $(\mathrm{OMS})^{17,23,24}$.

Menopausia prematura. Es la ocurrencia de la menopausia antes de los 40 años de edad, lo que sucede en alrededor del $1 \%$ de las mujeres. Estas mujeres tienen un riesgo elevado de sufrir osteoporosis y enfermedad cardiovascular $22,23,25$.

\section{Etapas de climaterio}

Según los criterios del Grupo de Trabajo de los Estadíos del Envejecimiento Reproductivo+10 (STRAW+10: Stages of Reproductive Aging Workshop) ${ }^{26}$ delimitan en

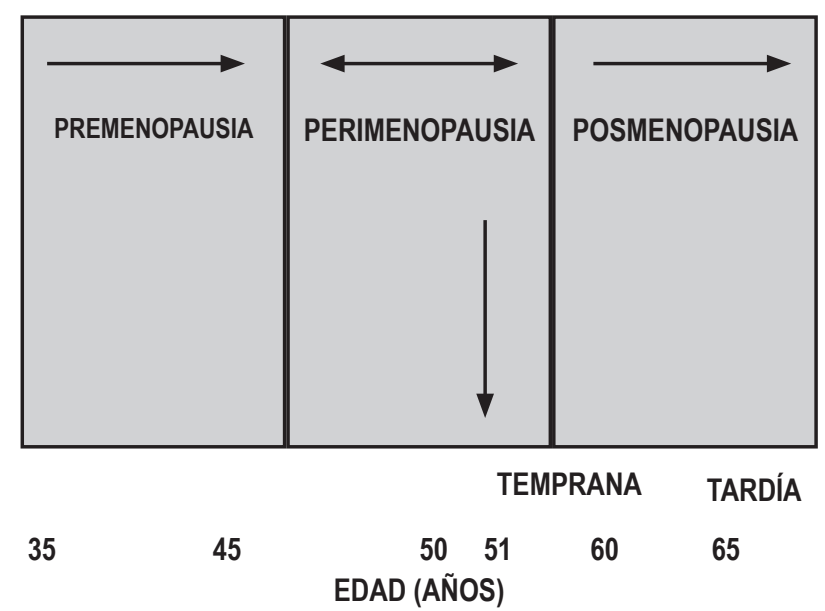

subetapas como son: Estado reproductivo, transición menopáusica o perimenopausia y postmenopausia mediante criterios clínicos y hormonales. Clásicamente se dividen en: premenopausia, perimenopausia y postmenopausia.

ETIOLOGÍA. La menopausia natural, es consecuencia de la atresia total de los folículos ováricos, con disminución de niveles de estrógenos e inhibina $\mathrm{B}$, incremento de la secreción de hormonas gonadotropinas hipofisiarias $\mathrm{FSH}$ y $\mathrm{LH}^{27}$.

FISIOPATOLOGÍA. La pérdida de la función ovárica suele ser un proceso gradual, que se produce en varios años, en promedio dura entre 4 a 6 años; en esta etapa, la función ovárica va fluctuando.

En este periodo, los niveles de estradiol son muy bajos, se produce un gran aumento de la $\mathrm{FSH}$, en menor medida de la LH, de tal forma que el cociente FSH/LH se invierte. Los estrógenos secretados por los ovarios después de la menopausia son insignificantes; sin embargo, todas las mujeres siguen teniendo concentraciones perceptibles de estradiol y estrona.

La estrona es el estrógeno más importante en la posmenopausia; se produce por la conversión periférica de la androstenediona (producida en la corteza suprarrenal y en el ovario), el cual se aromatiza en estrógenos, principalmente en el tejido adiposo.

Las obesas suelen tener una alta concentración de estrógenos circulantes; por tal motivo, están en peligro de sufrir cáncer de endometrio. En contraste, las mujeres delgadas experimentan disminución de los estrógenos circulantes, estando más expuestas a la osteoporosis ${ }^{21}$.

\section{EPIDEMIOLOGÍA}

A nivel mundial, la pirámide poblacional ha cambiado en las últimas décadas, incrementándose significativamente 
la esperanza de vida de la mujer. Las estadísticas de la Organización Mundial de la Salud muestran que en pocos años se alcanzará la cifra mundial de 750 millones de mujeres posmenopáusicas. La longevidad actual de la mujer posmenopáusica puede constituirse en más de $33 \%$ de la vida de la población femenina ${ }^{28}$.

En el Perú ${ }^{21,29}$, la esperanza de vida al nacer (EVN) de la mujer para el quinquenio 2010- 2015 se estima en 76,84 años y se ha previsto que se incrementará a 81,94 para el quinquenio 2045-2050.

La edad media de la menopausia varía según la ubicación

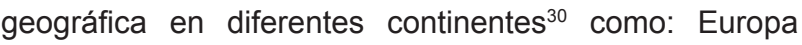
50,1- 52,8 años; América del Norte 50,5 a 51,4 años; América Latina 43,8 a 53 años, y Asia 42,1 a 49,5 años. En Latinoamérica, según estudio REDLINC ${ }^{30,31}$, la edad promedio de menopausia natural es de 48,6 años ( rango 43,8 a 53 )

En el Perú la edad promedio de la menopausia es de 47 años ${ }^{21,31}$. En Lima (150 msnm) es 47,1 años ${ }^{21,23,32-34}$, Cuzco (3400 msnm) 45 años, Cerro de Pasco (4340 msnm) 42 años $^{23,32,33}$

La prevalencia de síntomas vasomotores relacionados con la menopausia varía según la región geográfica: $74 \%$ de mujeres en Europa; 36-50\% de mujeres en América del Norte; $45-69 \%$ de mujeres en América Latina y $22-63 \%$ de mujeres en Asia ${ }^{27}$.

\section{FACTORES DE RIESGO ASOCIADOS ${ }^{35-37}$}

a. Incremento de edad.

b. Medio ambiente: altura ( $>2000 \mathrm{msnm}$ ) tienen mayor riesgo de hacer Menopausia temprana.

c. Estilos de Vida: El tabaco produce un adelanto de aproximadamente 1,5 años.

Falta de ejercicios, historia materna de síntomas vasomotores.

d. Menopausia temprana: Nuliparidad, uso de antidepresivos, quimioterápicos, radiación pélvica, bajo índice de masa corporal.

e. Menopausia tardía: multiparidad, incremento de masa corporal

f. Factores hereditarios: Factores familiares.

\section{CUADRO CLÍNICO}

\section{Síntomas y signos:}

Mediante la escala de puntuación menopáusica $(\mathrm{MRS})^{39,40}$ las manifestaciones clínicas en climaterio se pueden agrupar en 3 dimensiones o subescalas siguientes: somático-vegetativa, psicológica y urogenital (Tabla 1 ).
En la dimensión somático-vegetativa, se incluyen a los sofocos o bochornos y sudoración profusa; molestias cardíacas, como palpitaciones o bradicardias, consciencia no habitual de los latidos cardíacos; trastornos del sueño, como dificultad para dormir, despertarse con facilidad y pronto; y molestias músculo esqueléticas, como dolores en las articulaciones.

a) La dimensión psicológica, comprenden el estado de ánimo depresivo, como: sentirse deprimida, triste, a punto de llorar, falta de ímpetu, cambios en el estado de ánimo; irritabilidad, (sentirse nerviosa, agresiva); ansiedad (inquietud interna, sensación de pánico); y cansancio físico mental (menor rendimiento, problemas de memoria y menor concentración).

b) La dimensión urogenital define la presencia y severidad de problemas sexuales, como son la alteración de líbido/deseo, de la actividad y satisfacción sexual; problemas de vejiga, como dificultad a la hora de orinar, mayor necesidad de orinar e incontinencia vesical; y sequedad vaginal, que comprenden: sensación de sequedad o escozor en la vagina, dificultad en la relación sexual.

\section{DIAGNÓSTICO}

\section{Criterios de Diagnóstico:}

El diagnóstico es básicamente clínico. Se realizará mediante la historia clínica donde se hará especial énfasis en los siguientes aspectos ${ }^{21}$ :

a) Anamnesis: incluirá motivo de consulta y los antecedentes personales y familiares, identificar la presencia de factores de riesgo para la enfermedad cardiovascular, osteoporosis, cáncer genital y de mamas.

b) La evaluación de la calidad de vida se debe centrar, principalmente, en la percepción de la paciente sobre su estado de salud, siendo ella partícipe activa en este proceso. Para ello se utilizará la Escala de Puntuación Menopáusica (MRS) que es un instrumento específico para evaluar la sintomatología climatérica ${ }^{39-41}$.

c) Examen físico general y ginecológico, incluido mamas. Evaluar funciones vitales. Medición de talla, peso para calcular el índice de masa corporal y circunferencia de cintura. Este último sirve para expresar riesgo aterogénico cuando los valores sobrepasan los 88 centímetros.

d) Consignar el diagnóstico clínico certero es valioso lo que nos permitirá establecer las distintas etapas del climaterio: Premenopausia, perimenopausia y postmenopausia (temprana y tardía) ${ }^{21}$. 


\section{EXÁMENES AUXILIARES}

\section{De Patología Clínica}

Los exámenes de laboratorio clínico serán indicados de forma individualizada ${ }^{4}$.

La indicación del perfil hormonal sólo se justifica en aquellas mujeres que presenten una menopausia precoz o en casos muy específicos.

Exámenes Complementarios indispensables: Hemograma, glicemia y perfil lipídico. Este último debe realizarse una vez al año en las mujeres posmenopáusicas y si existe hiperlipidemia cada 6 meses. El perfil hepático (TGP, TGO, fosfatasa alcalina y gamma glutamil) debe indicarse para iniciar la terapia hormonal de menopausia (THM) a criterio médico y puede repetirse cada 6 meses si fuera necesario.

\section{De Imágenes}

Ultrasonografía. La ultrasonografía transvaginal con o sin doppler se indicará para: Iniciar la terapia hormonal de menopausia, control del endometrio durante y después de la THM, diagnosticar otras afecciones ginecológicas (pólipos endometriales, masas pélvicas, hiperplasias endometriales y otras). Se debe tener en cuenta el grosor endometrial $\geq$ de $5 \mathrm{~mm}$ en la mujer posmenopáusica para el diagnóstico presuntivo de anomalías endometriales y decidir la realización de la biopsia endometrial cuando se justifique ${ }^{21}$.

\section{Exámenes especializados complementarios}

a) Mamografía. Es necesario realizar una mamografía de base entre los 35 y 40 años de edad, las subsiguientes cada uno o dos años desde los 40 hasta los 49 años de edad y anualmente a partir de los 50 años de edad ${ }^{21}$. Si una mujer no ha tenido familiares con cáncer de mama, debe practicarse su primera mamografía a partir de los 40 años y repetirla anualmente, mientras que si hay antecedentes familiares de este mal, la mamografía debe realizarse a partir de los 35 años ${ }^{21}$.

La Ecografía de mamas permite distinguir entre mamas sólidas y quísticas.

b) Densitometría ósea. Estima el contenido mineral óseo (para establecer el riesgo de fractura) el método ideal es la absorciometría dual rayos-x (DEXA $)^{42}$. Es una prueba que sirve para: evaluar la salud ósea, llevar el control de la pérdida ósea, diagnosticar la osteoporosis, evaluar los resultados del tratamiento. El contenido mineral óseo (CMO) se expresa como: Puntaje T (T-Score) que resulta de comparar a la paciente con el momento en que se origina la mayor concentración del mineral en el hueso en columna lumbar, cuello femoral o cadera total.

c) Se puede utilizar el instrumento ORAl ${ }^{43}$ (Osteoporosis RiskAssessment Instrument) para identificar a aquellas pacientes mayores de 45 años con alta sospecha de osteoporosis que requerirán una densitometría ósea que confirme el diagnóstico, siendo el corte 8 puntos.

Tabla 1. Escala de puntuación en menopausia (MRS)

Con el objeto de conocer sus molestias climatéricas le solicitamos responder el siguiente cuestionario marcando el casillero correspondiente. ¿Cuál de las siguientes molestias siente en la actualidad y con qué intensidad?

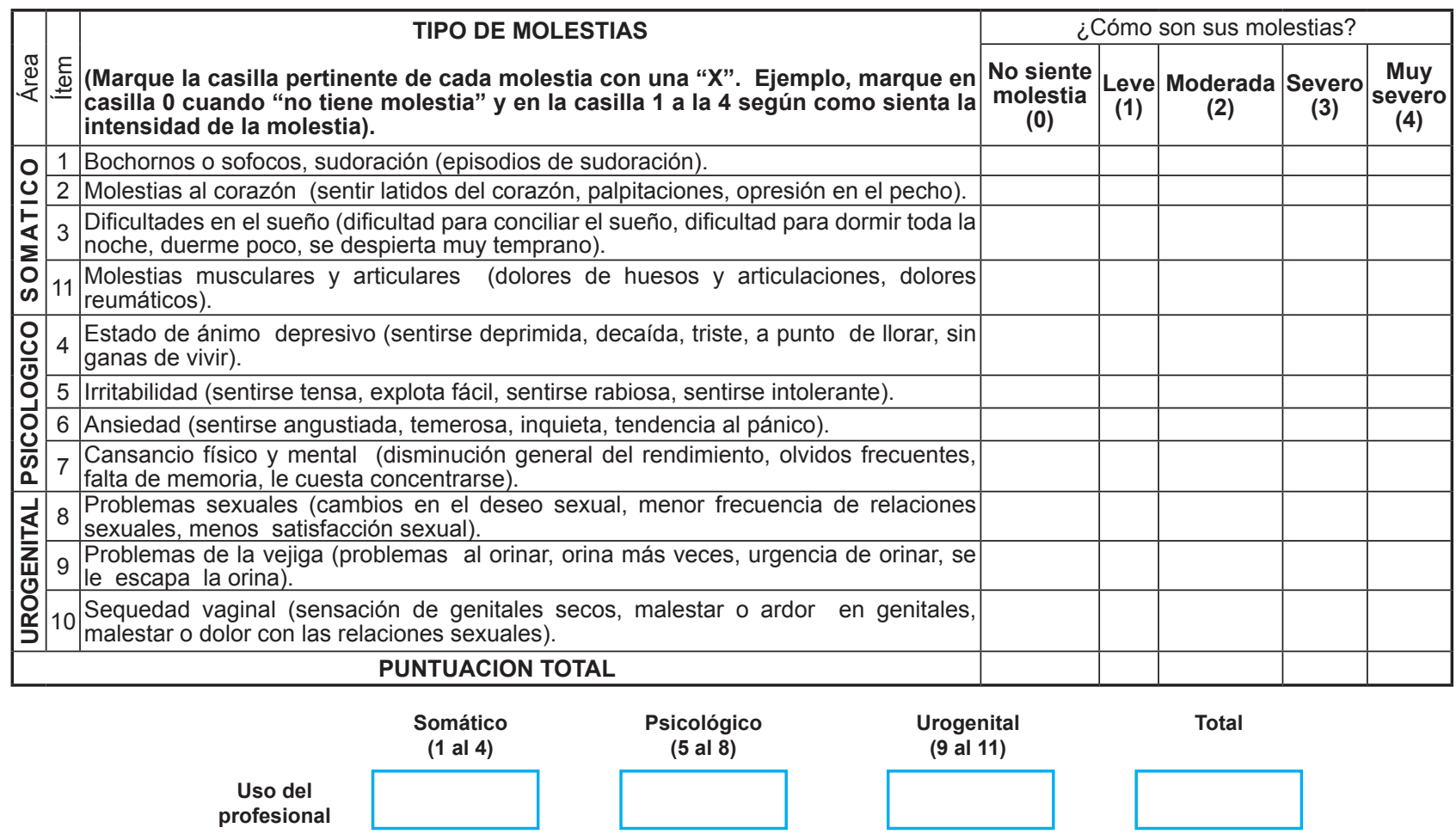


d) Otra opción es el FRAX ${ }^{44}$ (Fracture Risk Assessment Tool), una herramienta de evaluación de riesgo de fractura desarrollada por la Organización Mundial de la Salud.

\section{MANEJO INTEGRAL DURANTE LA ETAPA DEL CLIMATERIO}

\section{Medidas generales y preventivas}

Debe fomentarse la práctica de estilos de vida saludables, como ejercicio físico regular, control de peso, abandono de tabaquismo o bebidas alcohólicas y alimentación rica en calcio ${ }^{21,41}$.

Cualquier actividad física es mejor que permanecer sedentaria. El ejercicio regular reduce la mortalidad cardiovascular y total, mejor metabolismo, armonía física, fuerza muscular, cognición y calidad de vida son observadas en personas físicamente activas. Además, mejora los trastornos del sueño, regulariza el tránsito intestinal, disminuye en general los síntomas vasomotores y el estrés.

Los estudios muestran que en las mujeres postmenopáusicas los ejercicios aeróbicos y los de resistencia son efectivos para aumentar la densidad mineral ósea en la columna lumbar ${ }^{21}$.

\section{Terapéutica}

Se determina la necesidad de terapia en pacientes con compromiso de calidad de vida severo: definido según la escala MRS a un puntaje mayor a 16 puntos o como compromiso grave en un dominio determinado: Psicológico > a 6; Somático > a 8 y Urogenital > a 4 puntos ${ }^{21,41}$.

Antes de iniciarse cualquier terapia, debe evaluarse la presencia de contraindicaciones. Los estrógenos sin progesterona asociada generan un aumento de riesgo de cáncer de endometrio; las progestinas neutralizan este mayor riesgo. En relación con el tejido mamario, nuevos estudios no muestran una incidencia significativa en el aumento de riesgo de cáncer de mama.

La terapia hormonal oral debido a su efecto proinflamatorio y procoagulante por su paso hepático tiene un riesgo aumentado de trombosis. Por tal motivo se recomienda la vía transdérmica en mujeres con: obesidad (IMC $>30 \mathrm{Kg} / \mathrm{m}^{2}$ ), hipertensión arterial crónica, diabetes mellitus, litiasis biliar, hipertrigliceridemia, disfunción hepática crónica o antecedentes de tromboembolia por no modificar los parámetros de coagulación. Sin embargo, la vía transdérmica también tiene contraindicaciones como cáncer de mama, cáncer de endometrio, sangrado inexplicado y relativas (lupus, porfiria)

\section{INDICACIONES DE TERAPIA HORMONAL DE MENOPAUSIA (THM)}

La administración de THM de manera individualizada ${ }^{21,45-49}$ Las indicaciones de THM son las siguientes:

a. Tratamiento de los síntomas vasomotores de la menopausia.

b. Tratamiento del síndrome genitourinario de la menopausia.

c. Prevención de la osteoporosis en las mujeres con alto riesgo de fractura osteoporótica que no pueden tolerar los medicamentos preventivos estándar.

d. Menopausia prematura.

\section{CONTRAINDICACIONES DE THM ${ }^{21,45-49}$}

Absolutas:

Sangrado vaginal sin explicación

Disfunción o enfermedad hepática

Historia de trombosis venosa profunda o embolia pulmonar Trastorno de coagulación conocido o trombofilia (la presentación transdérmica puede ser una opción en algunas mujeres con riesgo elevado de trombosis venosa) Hipertensión no tratada

Historia de cáncer de mama, endometrio u otra neoplasia estrógeno-dependiente

Hipersensibilidad conocida a terapia hormonal

Historia de enfermedad coronaria, ACV o AIT.

Relativas: Triglicéridos altos (superiores a $400 \mathrm{mg} / \mathrm{dL}$ ). Enfermedad de la vesícula biliar (deben evitarse los estrógenos orales pero el estrógeno transdérmico puede ser una opción).

\section{ELECCIÓN DE LA TERAPIA HORMONAL EN MENOPAUSIA}

La THM de elección en mujeres sanas, perimenopáusicas y posmenopáusicas dentro de los 10 años de ocurrencia de su menopausia (o con edad $<60$ años), que presenten síntomas vasomotores moderados a severos, mujeres sintomáticas con alto riesgo de osteoporosis, y mujeres que han experimentado una falla ovárica prematura, considerado como ventana de oportunidad terapéutica ${ }^{45,46,50}$.

Para elegir el tratamiento más adecuado se requieren al menos dos disquisiciones mayores: distinguir a las mujeres que tienen contraindicaciones de terapia oral y aquellas que tienen útero de las que no la tienen. 
En mujeres sin contraindicaciones de terapia oral, disponemos las siguientes hormonas:

Estrógenos: Valerato de Estradiol 1mg/dia, 17-B-estradiol $1 \mathrm{mg} /$ día o estrógenos conjugados 0,3-0,45 mg/día.

Vía transdérmica en pacientes con contraindicaciones de vía oral, se utiliza: Estradiol gel 0,5 a 1,5mg/día o parche $0,5 \mathrm{mg} / \mathrm{día}$.

Si la mujer tiene útero es imprescindible la adición de una progestina: cíclica (10 a 14 días) o continua.

Progestinas: Se encuentra en presentaciones orales, parenteral, transdérmicas, endoceptivas. Opciones orales: Progesterona micronizada: continuo: $100 \mathrm{mg} /$ día o cíclico: 200 mg/día por 10 a 14 días. Drospirenona $2 \mathrm{mg} /$ día. Didrogesterona $5 \mathrm{mg} /$ día. Dienogest $2 \mathrm{mg} /$ día. Acetato de Medroxiprogesterona $5 \mathrm{mg} /$ día

Opciones transdérmica: Levonorgestrel 10 ug /día. Noretisterona 250ug/día

Opción endoceptiva: (SIU o DIU) Levonorgestrel 20ug. Liberados/24 hrs

Cominaciones de Estrógenos + Progestinas continuas: $17-B-e s t r a d i o l ~ 1 \mathrm{mg}+$ drosperinona $2 \mathrm{mg}$. Estradiol $1 \mathrm{mg}+$ didrogesterona $5 \mathrm{mg}$. Valerato de estradiol $1 \mathrm{mg}+$ dienogest $2 \mathrm{mg}$

Tibolona: A dosis 2,5 mg/día o 1,25 mg/día.

Estrógeno equino conjugado + Bazedoxifeno: Para mujeres con problemas de sensibilidad en las mamas, densidad mamaria, o sangrado uterino con THM convencional. Dosis: EC 0,45 mg/día + BZA 20mg/día

Ospemifeno: SERM $60 \mathrm{mg} / \mathrm{día}$, para tratamiento de síntomas genitourinarios de la menopausia, incluyendo sequedad vaginal y dispareunia, entre mujeres que prefieren tratamiento oral.

\section{ALTERNATIVAS DE LA THM}

Síndrome genitourinario de la menopausia ${ }^{51}$. La primera línea de tratamiento para las manifestaciones vaginales del SGM son los hidratantes (evidencia IA) y lubricantes vaginales (evidencia IIB). Cuando estas medidas no son suficientes, o en casos moderados 0 intensos, el tratamiento de elección son los estrógenos locales como: estradiol, estriol y promestrieno (evidencia IA); y éstas, no requieren la administración conjunta con gestágenos ni la realización de controles periódicos del endometrio; se presentan en crema, óvulos, comprimidos y anillos vaginales, con diferentes estrógenos y dosis; y se recomienda la aplicación de cremas tres veces por semana, óvulos y comprimidos dos veces por semana, de preferencia por la noche para comodidad de la usuaria. Si coexisten síntomas vasomotores que afectan la calidad de vida, la indicación es el tratamiento hormonal sistémico (evidencia IA). Actualmente, se dispone de ospemifeno (evidencia IA), un modulador selectivo de los receptores vaginales de estrógenos (SERM), aprobado en Europa para el tratamiento de los síntomas moderados o graves en mujeres postmenopáusicas que no cumplen los requisitos para recibir estrógenos vaginales. Otros posibles tratamientos del SGM son el láser y la radiofrecuencia.

Bochornos. La terapia hormonal en la menopausia es la primera elección para mitigar los bochornos, principalmente a bajas dosis o ultrabaja dosis. Si la THM está contraindicado o no se desea para el tratamiento de SVM, los inhibidores selectivos de la recaptación de serotonina y los inhibidores de la recaptación de serotonina-norepinefrina como antidepresivos son de primera línea en mujeres que no pueden tomar estrógenos $41,46,52$.

Paroxetina. Dosis: 7,5mg/día, al acostarse. Venlafaxina. Dosis: $37,5 \mathrm{mg} / \mathrm{día}$.

Desvenfalaxina. Dosis: 100mg/día. Gabapentina (600-900 $\mathrm{mg} / \mathrm{día})$; Clonidina (0,1 mg/día). Citalopram, Escitalopram son también efectivos. Sertralina y Fluoxetina tan bien son alternativas elegibles.

Osteoporosis en la postmenopausia. Todas las mujeres postmenopáusicas deberían ser incentivadas a practicar cambios en el estilo de vida que reduzcan el riesgo de pérdida ósea y de fractura osteoporótica: mantenimiento de un peso saludable, dieta balanceada, adecuada ingesta de calcio y vitamina D, ejercicio apropiado, evitar el cigarrillo y el excesivo consumo de alcohol y utilizar medidas para prevenir las caídas. Un adecuado consumo de cálcio y vitamina $D$ es fundamental en el mantenimiento de la salud ósea y es reconocido como un componente importante en el tratamiento farmacológico. El consumo recomendado de calcio en adultos $\geq 50$ años, es de $1200 \mathrm{mg} /$ día y 800 a 1000 UI/ día de vitamina D (NAMS, NOF) $41,45,46$.

El tratamiento farmacológico son con drogas antiresortivas:

1. Terapia hormonal de la menopausia, incluyendo tibolona, puede ser indicada en mujeres postmenopáusicas con riesgo de fractura menores de 60 años o dentro de los 10 años de la menopausia.

2. Raloxifeno: $60 \mathrm{mg} /$ día vía oral. Es un SERM para la prevención y tratamiento de la Osteoporosis ${ }^{53}$. Hay que considerar los riesgos/beneficios extra esqueléticos (aumento de sofocos, riesgos de trombosis profunda y producción de calambres)

3. Bifosfonatos ${ }^{53}$ entre ellos tenemos disponibles:

a) Alendronato. Preventivo a dosis 5mg/día. Terapéutico $10 \mathrm{mg} /$ día o $70 \mathrm{mg}$ semanal.

b) Ibandronato. Dosis oral $150 \mathrm{mg} / \mathrm{mes}$. Intravenoso $3 \mathrm{mg} / 3 \mathrm{meses}$. 
c) Risedronato. Dosis oral: $5 \mathrm{mg} / \mathrm{día}, 35 \mathrm{mg} / \mathrm{semana}$, $150 \mathrm{mg} / \mathrm{mes}$.

d) Acido zolendrónico. Dosis: $5 \mathrm{mg} /$ anual endovenoso.

SEGUIMIENTO. Incluyen nueva aplicación de escala de puntuación menopáusica, examen clínico, control de comorbilidades, ajustes de THM y no hormonal. Para el seguimiento del climaterio se debe separar a la población objetivo en 2 grupos: a) Mujeres usuarias de THM: control cada 3 meses hasta controlar los síntomas, luego una vez al año, y b) Mujeres no usuarias de THM control anual.

Financiamiento: Autofinanciado.

Conflicto de interés: El autor declara no tener conflicto de interés alguno.

\section{REFERENCIAS BIBLIOGRÁFICAS}

1. Ayala-Peralta F. Introducción al simposio manejo terapéutico en el climaterio y menopausia, evidencias actuales. Visión holística en manejo integral durante el climaterio. Rev Peru Ginecol Obstet.2018;64(1): 27-29.

2. American College of Obstetricians and Gynecologists (ACOG). Practice Bulletin No. 141: management of menopausal symptoms. Obstet Gynecol. 2014 Jan;123(1):202-16. DOI:10.1097/01. AOG.0000441353.20693.78. Correction can be found in Obstet Gynecol. 2016;127(1):166. DOI:10.1097/ AOG.0000000000001230.

3. Lopera-Valle JS, Parada-Pérez AM, Martínez-Sánchez LM,Jaramillo-Jaramillo LI; Rojas-Jiménez S. Calidad de vida en la menopausia, un reto en la práctica clínica. Revista Cubana de Medicina General Integral. 2016; 32(3).

4. Skjærvo GR, Roskaft E. Menopause: No support for an evolutionary explanation among historical Norwegians. Exp Gerontol. 2013;48(4):408-13.

5. Coronado PJ, Sánchez-Borrego R, Ruiz MA, Baquedano L, Sánchez S, Argudo C, et al. Psychometric attributes of the Cervantes short-form questionnaire for measuring healthrelated quality of life in menopausal women. Maturitas. 2016;84:55-62.

6. Jenabi E, Shobeiri F, Hazavehei SM, Roshanaei G. Assessment of Questionnaire Measuring Quality of Life in Menopausal Women: A Systematic Review. Oman Med J. 2015;30(3):151-6.

7. Lwow F, Jedrzejuk D, Dunajska K, Milewicz A, Szmigiero L. Cardiovascular disease risk factors associated with low level of physical activity in postmenopausal Polish women. GynecolEndocrinol. 2013;29(7):683-6.

8. Lizcano F, Guzmán G. Estrogen Deficiency and the Origin of Obesity during Menopause. Biomed Res Int. 2014;2014:1-12.

9. Kwak EK, Park HS, Kang NM. Menopause Knowledge, Attitude, Symptom and Management among Midlife Employed Women. J Menopausal Med. 2014;20(3):118-25

10. Comhaire FH, Depypere HT. Hormones, herbal preparations and nutriceuticals for a better life after the menopause: part I. Climacteric. 2015;18(3):358-63.

11. Comhaire FH, Depypere HT. Hormones, herbal preparations and nutriceuticals fora better life after the menopause: part II. Climacteric. 2015;18(3):364-71.

12. Hodis HN Mack WJ. Hormone replacement therapy and the association with coronary heart disease and overall mortality:
Clinical application of the timing hypothesis. J Steroid Biochem Mol Biol. 2013 Jul 9. pii: S0960-0760(13)00126-X. doi: 10.1016/j.jsbmb.2013.06.011.

13. Fenton A, Pany N. Hormone therapy and cardiovascular disease-are we back to the beginning? Climacteric. 2015;18(4):437-8. doi.org/10.3109/13697137.2015.1057958.

14. The North American Menopause Society. The 2017 hormone therapy position statement of The North American Menopause Society. Menopause. 2017;24(7):1-26.DOI: 10.1097/GME.0000000000000921.

15. Stuenkel CA, Davis SR, Gompel A, Lumsden MA, Murad $\mathrm{MH}$, Pinkerton JV, Santen RJ. Treatment of symptoms of the menopause: An Endocrine Society Clinical Practice Guideline. J Clin Endocrinol Metab. 2015;100(11):3975-4011. doi: 10.1210/jc.2015-2236.

16. Ayala-Peralta FD, Ayala R, Ayala D. Climaterio y menopausia: aporte de 70 años de vida institucional. Rev Peru Ginecol Obstet. 2017;63(3):455-62.

17. Parra $M$, Lagos $N$, Levancini $M$, Villarroel $M$, Pizarro $E$, Vanhauwaert P, et al. Guía Clínica. Menopausia y terapia hormonal de la menopausia Las recomendaciones 2018 de la Unidad de Endocrinología Ginecológica de Clínica Alemana de Santiago -Sociedad Italiana de la Menopausia y la Sociedad Chilena de Endocrinología Ginecológica. Rev Chil Obstet Ginecol 2018; 83(5): 527 - 550

18. Pacheco J, Salvador J. Conocimiento y actitudes de la mujer peruana sobre el climaterio y menopausia. Revista Sociedad Peruana de Obstetricia y Ginecología 1998; 44 3: 225-230.

19. Utian WH. The International Menopause Society menopauserelated terminology definitions. Climateric 1999;2:284-6.

20. Montaño R, Urrutia J, Schepeler M. Conceptos y definiciones en climaterio y menopausia. En: Consenso en Climaterio 2001. Sociedad Chilena del Climaterio. Santiago de Chile 2001;1: 9-12.

21. Ayala FD. Climaterio y menopausia. Perspectiva de manejo de salud femenina. Editorial CONCYTEC Perú. 1ra Edición. 2006

22. Sociedad Peruana del Climaterio. Información Básica sobre la menopausia. Guía de Práctica Clínica sobre el Climaterio y Menopausia. Perú 2008; 1: 33-39.

23. Rojas JA, Gonzales GF, Olavide JR, Pacora P. Atención del climaterio y menopausia en la mujer peruana. Diagnóstico 2013;52:80-99.

24. Sinha $P$, Kuruba N. Premature ovarían failure. J Obstet Gynaecol 2007;27:16-9.

25. Coulam CB, Adamson SC, Annegers JF. Incidence of premature ovarian failure. Obstet Gynecol 1986;67:604

26. Harlow SD, Gass M, Hall JE, Lobo R, Maki P, Rebar RW, el al for the STRAW+10 Collaborative Group. Executive summary of the Stages of Reproductive Aging Workshop+10: addressing the unfinished agenda of staging reproductive aging. Fertil Steril 2012;97:843-51.

27. Sociedad de Ginecología y Obstetricia de Buenos Aires (SOGIBA). Consenso de Climaterio: Rol actual de la terapia hormonal de menopausia y estrategias de prevención en la medicina del climaterio. Argentina,2018.

28. Villiers TJ, Pines A, Panay N, Gambacciani M, Archer DF et al Update 2013 International Menopause Society recommendations on menopausal hormone therapy and preventive strategies for midlife health. Climateric. 2013;16:316-37.

29. Instituto Nacional de Estadística e Informática. Perú: Estimaciones y proyecciones de la población 2010-2050. Boletín de Análisis Demográfico №36. Lima 2009.

30. Palacios S, Henderson VW, Siseles N, Tan D, Villaseca P. Age of menopause and impact of climateric symptoms by geographical region. Climateric 2010;13:419-428. 
31. Castelo-Branco C, Blümel JE, Chedraui P, Calle A,Bocanera $\mathrm{R}$, Depiano $\mathrm{E}$, et al. Age at menopause in Latin America. Menopause 2006;13:706-12.

32. Gonzales, GF. Estudio sobre la menopausia en el Perú. Acta Andina 1994; 3: 55-70.

33. Gonzales GF, Villena A. Age at menopause in central Andean Peruvian Women. Menopause 1997;4:32-38.

34. Gonzales GF, Villena A, De La Cruz D. Age of natural menopause among women in Lima City, Peru. International Journal of Gynecology \& Obstetrics 1997;57:69-72.

35. Blümel JE, Fica J, Chedraui $P$, Mezones-Holguín E, Zuñiga MC, Witis S, Vallejo MS, Tserotas K, Sánchez H, Onatra W, Ojeda E, Mostajo D, Monterrosa A, Lima S, Martino $M$, Hernández-Bueno JA, Gómez G, Espinoza MT, Flores D, Calle A, Bravo LM, Benítez Z, Bencosme A, Barón G, Aedo S; Collaborative Group for Research of the Climacteric in Latin America. Sedentary lifestyle in middle-aged women is associated with severe menopausal symptoms and obesity. Menopause. 2016;23:488-93. doi: 10.1097/ GME.0000000000000575.

36. Pokoradi AJ, Iversen L, Hannaford PC. Factors associated with age of onset and type of menopause in a cohort of UK women. Am J Obstet Gynecol. 2011;205:34.e1-13. doi: 10.1016/j.ajog.2011.02.059.

37. Tao X, Jiang A, Yin L, Li Y, Tao F, Hu H. Body mass index and age at natural menopause: a meta-analysis. Menopause. 2015 Apr;22:469-74. doi: 10.1097/GME.0000000000000324.

38. Blümel JE, Fica J, Chedraui $P$, Mezones-Holguín E, Zuñiga MC, Witis S, Vallejo MS, Tserotas K, Sánchez H, Onatra W, Ojeda E, Mostajo D, Monterrosa A, Lima S, Martino M, Hernández-Bueno JA, Gómez G, Espinoza MT, Flores D, Calle A, Bravo LM, Benítez Z, Bencosme A, Barón G, Aedo S; Collaborative Group for Research of the Climacteric in Latin America. Sedentary lifestyle in middle-aged women is associated with severe menopausal symptoms and obesity. Menopause. 2016;23:488-93. doi: 10.1097/ GME.0000000000000575.

39. Pokoradi AJ, Iversen L, Hannaford PC. Factors associated with age of onset and type of menopause in a cohort of UK women. Am J Obstet Gynecol. 2011;205:34.e1-13. doi: 10.1016/j.ajog.2011.02.059.

40. Tao X, Jiang A, Yin L, Li Y, Tao F, Hu H. Body mass index and age at natural menopause: a meta-analysis. Menopause. 2015 Apr;22:469-74. doi: 10.1097/GME.0000000000000324.

41. Monsalve C, Reyes V, Parra J, Chea R. Manejo terapéutico de la sintomatología climatérica. Rev Peru Ginecol Obstet. 2018;64(1):43-50.

42. NIH. Consensus Development Panel on Osteoporosis. J AMA 2001;285:78595.
43. SOCHEG. Instrumento ORAI para decidir densitometría. Disponible en:

http://www.socheg.cl/Anexo\%200RAI.pdf. Diciembre 18, 2017.

44. WHO. http://www.shef.ac.uk/FRAX/index_SP.htm .

45. NAMS. The 2017 hormone therapy position statement of The North American Menopause Society. Menopause. 2017;24(7):728-753.

46. The American College of Obstetricians and Gynecologists. Practice Bulletin $\mathrm{N}^{\circ}$ 141. Management of menopausal symptoms. Obstet Gynecol. 2014;123(1):202-214.

47. Harper-Harrison G, Shanahan MM. Hormone Replacement Therapy. [Updated 2018 Feb 26]. In: StatPearls [Internet]. Treasure Island (FL): StatPearls Publishing; 2018 Jan. Available from: https://www.ncbi.nlm.nih.gov/books/ NBK493191/

48. Kaunitz y Manson. Manejo de Síntomas de la Menopausia. The American College of Obstetricians and Gynecologists. Obstet Gynecol. 2015;126:859-76. www.greenjournal.org

49. de Villiers TJ, Stevenson JC. The WHI: the effect of hormone replacement therapy on fracture prevention. Climacteric.2012;15: 263-6.

50. The North American Menopause Society recommendations for clinical care of midlife women. Shifren JL, Gass ML;NAMS Recommendations for Clinical Care of Midlife Women Working Group. Menopause. 2014;21(10):1038-62. doi:10.1097/ GME.0000000000000319.

51. Palacios S, Cancelo Hidalgo MJ, González SP, Manubens $M$, Sánchez-Borrego R. Síndrome genitourinario de la menopausia: recomendaciones de la Sociedad Española de Ginecología y Obstetricia. Prog Obstet Ginecol 2019;62(2):141-148. DOI: 10.20960/j.pog.00182.

52. NAMS. Nonhormonal management of menopauseassociated vasomotor symptoms: 2015 position statement of The North American Menopause Society. Menopause. 2015;22(11):1155-1174.

53. NAMS. Management of osteoporosis in postmenopausal women: 2010 position statement of The North American Menopause Society. Menopause. 2010;17(1):25-

\section{Correspondencia:}

Félix Dasio Ayala Peralta.

Dirección: Jirón Maracaibo N²153-San Martín de Porres, LimaPerú

Correo: fayala1401@hotmail.com

Teléfono: 999227657 